\title{
Comparative study of single-nucleotide polymorphism array and next generation sequencing based strategies on triploid identification in preimplantation genetic diagnosis and screen
}

\author{
Jiawei Xu' ${ }^{1, *}$, Wenbin Niu, ${ }^{1, *}$, Zhaofeng Peng ${ }^{1}$, Xiao Bao ${ }^{1}$, Meixiang Zhang ${ }^{1}$, Linlin \\ Wang $^{1}$, Linqing Du ${ }^{1}$, Nan Zhang ${ }^{1}$, Yingpu Sun ${ }^{1}$ \\ ${ }^{1}$ The First Affiliated Hospital of Zhengzhou University, Centre for Reproductive Medicine, Zhengzhou, Henan 450000, China \\ *These authors have contributed equally to this work \\ Correspondence to: Yingpu Sun, email: syp2008@vip.sina.com \\ Keywords: triploidy, SNP array, next generation sequencing, MALBAC, preimplantation genetic diagnosis and screen \\ Received: September 23, 2016 \\ Accepted: October 14, 2016 \\ Published: November 09, 2016
}

\section{ABSTRACT}

Triploidy occurred about $2-3 \%$ in human pregnancies and contributed to approximately $15 \%$ of chromosomally caused human early miscarriage. It is essential for preimplantation genetic diagnosis and screen to distinct triploidy sensitively. Here, we performed comparative investigations between MALBAC-NGS and MDASNP array sensitivity on triploidy detection. Self-correction and reference-correction algorism were used to analyze the NGS data. We identified 5 triploid embryos in 1198 embryos of 218 PGD and PGS cycles using MDA-SNP array, the rate of tripoidy was 4.17\% in PGS and PGD patients. Our results indicated that the MDA-SNP array was sensitive to digyny and diandry triploidy, MALBAC-NGS combined with self and reference genome correction strategies analyze were not sensitive to detect triploidy. Our study demonstrated that triploidy occurred at 4.17\% in PGD and PGS, MDASNP array could successfully identify triploidy in PGD and PGS and genomic DNA. MALBAC-NGS combined with self and reference genome correction strategies were not sensitive to triploidy.

\section{INTRODUCTION}

Triploidy is an abnormal chromosome kayotype, which occurred about $2-3 \%$ in human pregnancies [1] and contributed to approximately $15 \%$ of chromosomally caused human early miscarriage. The biological mechanism of triploidy may be of either digyny (one more haploid got from mother) or diandry (one more haploid got from father), and digynic triploidy predominates in fetuses leading to about $50-60 \%$ of early triploidy spontaneous pregnancy loss [2-5]. Although genomic imprinting or whole genome gene expression disturbed resulted to triploidy often early spontaneous abortion, triploidy still occasionally could develop to fetal or newborn period with the birth of an abnormal fetus or infants [6]. And assessment of embryonic phenotype with parental origin showed no correlation between the phenotype of the embryo and parental origin of the extra haploid set in triploid pregnancy [7].

Three pronucleis (3PN) embryo formation is common during in vitro fertilization (IVF), and is believed that polyspermic fertilization or oocyte-derived meiotic failure lead to triploid $[8,9]$. Those patients with advanced maternal age or severe sperm abnormalities significant increased the incidence of triploidy fertilization during IVF [10]. Intracytoplasmic sperm injection (ICSI) essentially excluded dispermic triploidy but cannot prevent oocyteinduced triploid, such as the second polar boby exhausted failure [11]. It had been reported that triploid embryos negatively associated with IVF pregnancy outcome [12]. ICSI was recommended in patients who would undergo treatment of premimplantation genetic diagnosis and screen (PGD/S) [13]. Previous study suggests that 3PN embryos formation in ICSI treatment was mainly due to nonextrusion of the second polar body [14], severe sperm abnormalities, oocyte aging and women who are high responders to gonadotropins may also contribute to this process $[9,10,15]$. Sometimes $3 \mathrm{PN}$ embryos could not be observed due to fusion of pronuclear during IVF, hence, it was essential to consideration the formation and detectable of triploid embryos during PGD/S. 
PGD/S was used for chromosome structure abnormality carrier patients, single gene mutation carrier patients, advanced maternal age couples, recurrent implantation failure and recurrent miscarriage patients to screen embryos genetic condition prior to transfer [16], along with single cell amplification technology developed, genome-wide technologies such as array-CGH, singlenucleotide polymorphism (SNP) array and next generation sequencing (NGS) are applied to PGD/S [17-19]. All of these three methods can successfully detect chromosome imbalances in embryos, also providing extra benefit of simultaneous aneuploidy screen of all 24 chromosomes $[20,21]$. NGS was becoming more and more popular in $\mathrm{PGD} / \mathrm{S}$ for lower cost, higher resolution and providing opportunity to simultaneously analyze single-gene disorders and genome-wide chromosome imbalance diagnosis and screen [22-24]. MDA and MALBAC, two main powerful single cell amplification methods were widely used in $\mathrm{PGD} / \mathrm{S}$, have performed high coverage and accuracy of whole genome $[25,26]$. SNP based noninvasive prenatal testing (NIPT) have distinguished triploid pregnancy, and NGS based NIPT together with NATUS algorithm analyzing sequencing data identified triploid pregnancy using cfDNA in maternal blood [27, 28]. Although $\mathrm{PGD} / \mathrm{S}$ has increased the pregnancy rate, no studies explore the ability on triploid embryos detection between SNP array and NGS technologies in PGD/S.

Here in our study, we systematically compared SNP array and NGS on detection chromosome deletion, duplication, uniparental disomy, mosaic and triploidy. We firstly identified triploid embryos using SNP array and compared it with NGS during PGD/S, our results indicated that present NGS based PGD/S procedures were unable to detect triploid embryos but SNP array can successfully distinguished triploidy.

\section{RESULTS}

\section{Triploid embryos during PGD/S using MDA- SNP array}

We have detected 5 triploid embryos in 1198 embryos of 218 PGD/S cycles using MDA-SNP array (Table 1 and Figure 1). The rate was $4.17 \%$, which is lower than previous reported human triploid pregnancy rate $2-3 \%$ [1]. These triploid embryos in 5 PGD cycles with three chromosome translocation and two robertsonian translocation. All the triploidy detected were arr (1-22) $\times 3$, $(\mathrm{X}) \times 3$ (Table 1$)$. We didn't detect any $\operatorname{arr}(1-22) \times 3, X Y Y$ and $\operatorname{arr}(1-22) \times 3$, XXY karyotype.

\section{Comparative MDA-SNP array and MALBAC- NGS during copy number variance screen}

We firstly compared the SNP array and NGS on detection copy number variance. We diluted DNA from previously known SNP karyotype missed abortion chorionic tissues then whole genome amplification using MALBAC. The amplification product were sequenced and analyzed, 46 samples previously known SNP array karyotype including duplication, deletion, mosaics and uniparental disomy (UPD), the results of SNP array and NGS were highly in accordance (Table 2). The NGS is sensitive to duplication, deletion and mosaics, but UPD couldn't be identified. Furthermore, NGS could identify more copy number variance than SNP array and is powerful on detection low percentage mosaics.

\section{Digyny and diandry triploidy detection using MDA-SNP array}

Our previous data detected triploidy in five PGD cycles, however, these only identified $\operatorname{arr}(1-22) \times 3,(\mathrm{X}) \times 3$, however, whether the arr $(1-22) \times 3,(X X Y) \times 1$ and $\operatorname{arr}(1-$ $22) \times 3,(X Y Y) \times 1$ could be detected or not in single cell lever using MDA based SNP array is still unknown. Here we system investigated the sensitivity of MDA based SNP array for the digyny and diandry triploidy on single cell lever. Our results showed MDA based SNP array could completely identify the arr $(1-22) \times 3,(X) \times 3$, arr $(1-22)$ $\times 3,(X X Y) \times 1$ and arr $(1-22) \times 3,(X Y Y) \times 1$ (Figure 2$)$, indicating that all types of triploidy would be detected during $\mathrm{PGD} / \mathrm{S}$.

\section{Digyny and diandry triploidy detection using MALBAC-NGS}

We selected 9 previous known triploids SNP array karotype to validate using NGS. The 9 triploid SNP array kayotype were shown in Supplementary Figure 1, one was amplification failure in our study. The basic information of 9 sample data was shown in Supplementary Table 1. After sequencing, we firstly using reference karyotype methods to analyzed the data, and we found that NGS could not detect triploid for it considered the triploid as normal diploid (Supplementary Table 2). Then we using self correction method to analyze the data, this could not detect the triploid karyotype (See Figure 3). The unique reads of NGS data were ranging from $1.23 \mathrm{M}$ to $4.84 \mathrm{Mb}$, both the low and high coverage didn't identify the triploidy in our study. Our results indicated that both self and reference correction may not be able to detect the triploid, mostly because both these methods calculated the reads of 24 chromosome falling into the continuous sliding widows on human reference genome.

\section{DISCUSSION}

Triploidy is an abnormal chromosome kayotype, which occurred at very low percent in human pregnancies, this is the first time comprehensive analyzed the triploidy detection using SNP array and NGS in PGD/S and missed abortion chorionic tissues. Our results indicated that the rate of tripoidy was $4.17 \%$ in $\mathrm{PGD} / \mathrm{S}$ patients. In our 
Table 1: Clinical characteristic of Triploid detected PGD/S cycles using SNP array

\begin{tabular}{|c|c|c|c|c|c|c|c|c|c|c|}
\hline ID & $\begin{array}{l}\text { F- } \\
\text { Age }\end{array}$ & $\mathbf{F}_{-} \mathbf{K}$ & M_Age & M_K & N(Oocyte) & N(MII) & Days & $\mathbf{E}_{-}$ & $\begin{array}{c}\text { E_- } \\
\text { Score }\end{array}$ & $\begin{array}{l}\text { Embryo SNP } \\
\text { Karyotype }\end{array}$ \\
\hline \multirow[t]{3}{*}{ Cycle 1} & 28 & $46, X X$ & 24 & $\begin{array}{c}46, X Y, t(4,7)(\mathrm{p} 1 \\
6, \mathrm{q} 22)\end{array}$ & 11 & 7 & 5 & 1 & $3 \mathrm{BB}$ & $\begin{array}{c}\operatorname{arr} 4 \mathrm{p}(\mathrm{p} 15.31 \rightarrow \mathrm{qter}) \times \\
3,7 \mathrm{p}(\mathrm{pter} \rightarrow \mathrm{q} 22.1) \times 1 \\
(\mathrm{X}) \times 2\end{array}$ \\
\hline & & & & & & & 5 & 2 & $3 \mathrm{BB}$ & Amplification Failure \\
\hline & & & & & & & 5 & 3 & 3BB & $\operatorname{arr}(1-22) \times 3,(X) \times 3$ \\
\hline \multirow[t]{4}{*}{ Cycle 2} & 27 & 46,XX & 27 & $\begin{array}{l}\text { 45,XY,rob(13;1 } \\
\text { 4)(q10;q10) }\end{array}$ & 9 & 6 & 5 & 1 & 3BC & $\operatorname{arr}(1-22) \times 3,(X) \times 3$ \\
\hline & & & & & & & 5 & 2 & $2 \mathrm{BB}$ & $\underset{21(\text { pter } \rightarrow \text { qter }) \times 1,(X) \times 2}{\operatorname{arr}}$ \\
\hline & & & & & & & 5 & 3 & $3 \mathrm{BB}$ & $\operatorname{arr}(1-22) \times 2,(X) \times 2$ \\
\hline & & & & & & & 5 & 4 & $4 \mathrm{BC}$ & $\operatorname{arr}(1-22) \times 2,(\mathrm{XY}) \times 1$ \\
\hline \multirow[t]{3}{*}{ Cycle 3} & 34 & $46, X X$ & 38 & $\begin{array}{l}45, X Y, \operatorname{rob}(14 ; 2 \\
1)(q 10 ; q 10)\end{array}$ & 16 & 16 & 5 & 1 & 3BB & $\operatorname{arr}(1-22) \times 3,(X) \times 3$ \\
\hline & & & & & & & 5 & 2 & $4 \mathrm{AB}$ & $\operatorname{arr}(1-22) \times 2,(X Y) \times 1$ \\
\hline & & & & & & & 5 & 3 & $6 \mathrm{BB}$ & $\operatorname{arr}(1-22) \times 2,(\mathrm{XY}) \times 1$ \\
\hline \multirow[t]{5}{*}{ Cycle 4} & 29 & $\begin{array}{l}46, X X, t(9,1 \\
7)(p 13, q 12)\end{array}$ & 31 & 46,XY & 21 & 19 & 5 & 1 & $3 \mathrm{BB}$ & $\operatorname{arr}(1-22) \times 2,(\mathrm{XY}) \times 1$ \\
\hline & & & & & & & 5 & 2 & $4 \mathrm{BB}$ & $\begin{array}{c}\text { arr } \\
9 \mathrm{q}(\mathrm{q} 13 \rightarrow \mathrm{qter}) \times 3,17 \mathrm{p}- \\
,(\mathrm{X}) \times 2\end{array}$ \\
\hline & & & & & & & 5 & 3 & $2 B B$ & $\operatorname{arr}(1-22) \times 3,(X) \times 3$ \\
\hline & & & & & & & 5 & 4 & $2 \mathrm{BB}$ & $\begin{array}{c}\operatorname{arr} 9(\text { pter } \rightarrow \text { qter }) \times 3,22 \\
(\text { pter } \rightarrow \text { qter }) \times 1 \\
17 \text { p- },(X Y) \times 1\end{array}$ \\
\hline & & & & & & & 5 & 5 & $2 \mathrm{BC}$ & $\begin{array}{c}\operatorname{arr} 19 \mathrm{p}(\mathrm{p} 13.2 \rightarrow \mathrm{pter}) \times \\
1,(\mathrm{X}) \times 2\end{array}$ \\
\hline \multirow[t]{6}{*}{ Cycle 5} & 27 & $\begin{array}{l}46, X X, t(11 ; 1 \\
5)(p 15.4 ; q 25)\end{array}$ & 27 & $46, X Y$ & 15 & 14 & 5 & 1 & $3 \mathrm{BB}$ & $\operatorname{arr}(1-22) \times 2,(X) \times 2$ \\
\hline & & & & & & & 5 & 2 & 2BB & $\operatorname{arr}(1-22) \times 3,(X) \times 3$ \\
\hline & & & & & & & 5 & 3 & $3 \mathrm{BB}$ & $\begin{array}{l}\operatorname{arr} 4 q(q 33 \rightarrow q \text { ter }) \times 1 \\
(X) \times 2\end{array}$ \\
\hline & & & & & & & 6 & 4 & $5 \mathrm{BB}$ & $\begin{array}{l}\text { arr } 16(\text { pter } \rightarrow \text { qter }) \times 1 \\
(X) \times 2\end{array}$ \\
\hline & & & & & & & 6 & 5 & $5 \mathrm{BB}$ & $\begin{array}{c}\operatorname{arr}(1-22) \times 2 \\
(\mathrm{X}) \times 2\end{array}$ \\
\hline & & & & & & & 6 & 6 & $6 \mathrm{BB}$ & $\operatorname{arr}(1-22) \times 2,(X) \times 2$ \\
\hline
\end{tabular}

5 cycles identified triploidy were shown from 218 cycles.

F_Age: Female age, F_K: Female karyotype, M_Age: Male age, M_K: Male karyotype, N(Oocyte): Number of retrieve oocyte, N(MII): Number of MII oocyte, E_ID: Embryo_ID, E_Score: Embryo Score.

study, we comprehensive compared the MDA based SNP array and MALBAC based NGS for triploid detection on single cell lever. And we concluded that SNP array could detect triploidy, while the present strategies of NGS are not sensitive to triploidy detection.

PGD and PGS were used to detect the aneuploidy and pathological gene carrier embryos and has been used 
Cycle 1-3
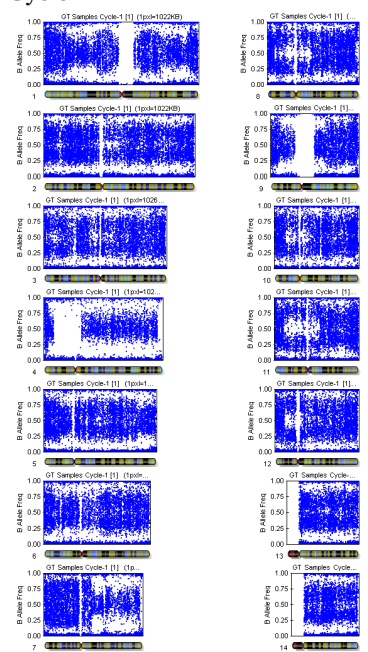

I.
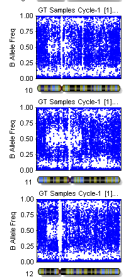

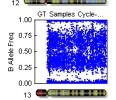

华

Cycle 3-1
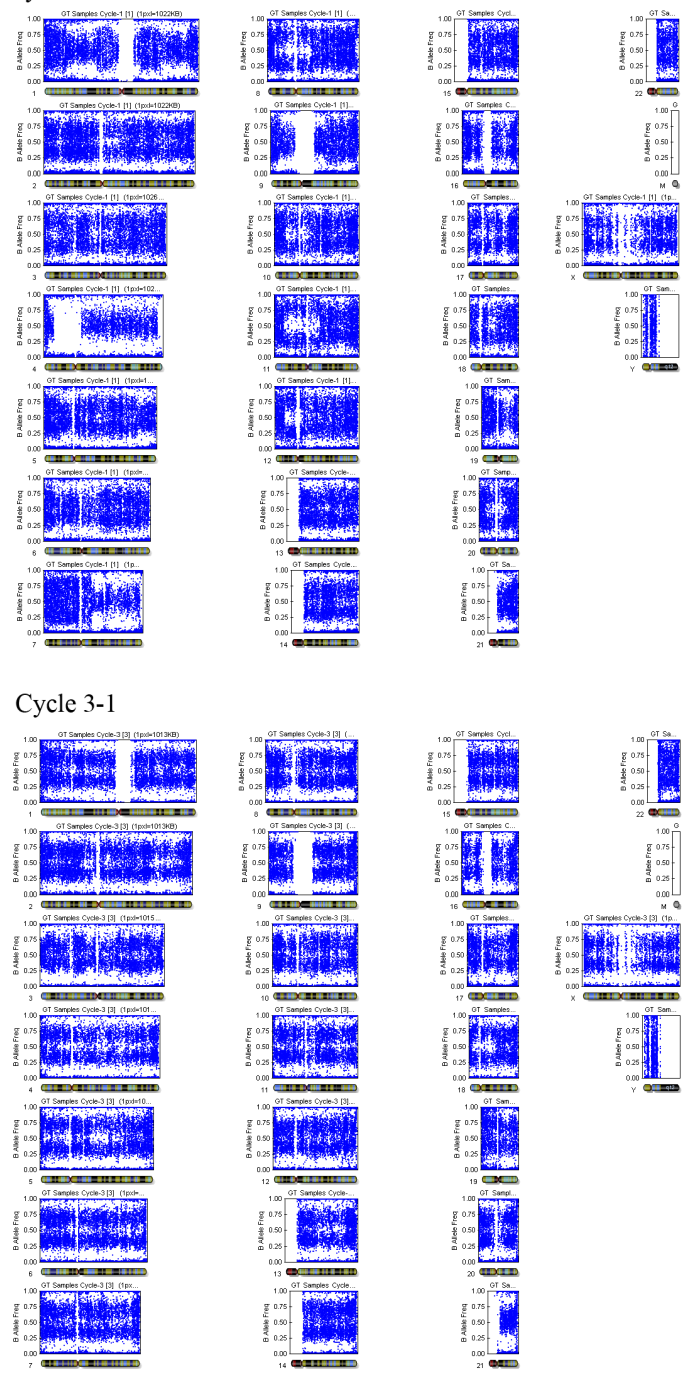

패표

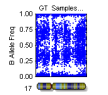

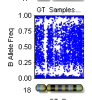

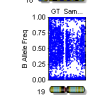

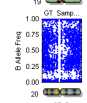

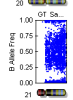

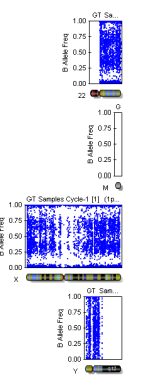

Cycle 2-1
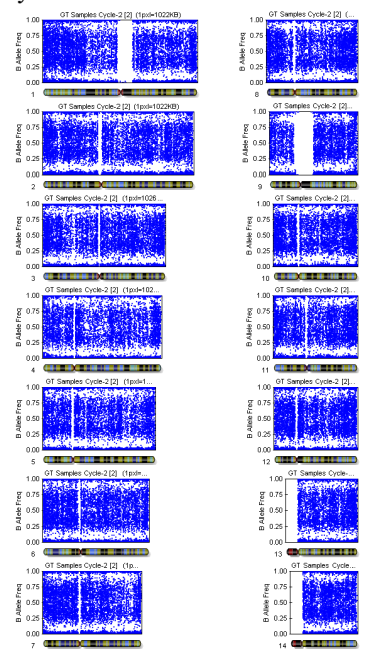

I.

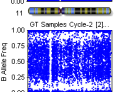

ix

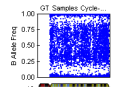

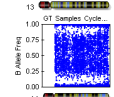

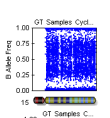

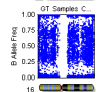

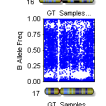

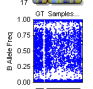

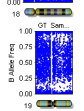

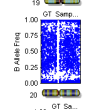

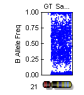

Cycle 4-3
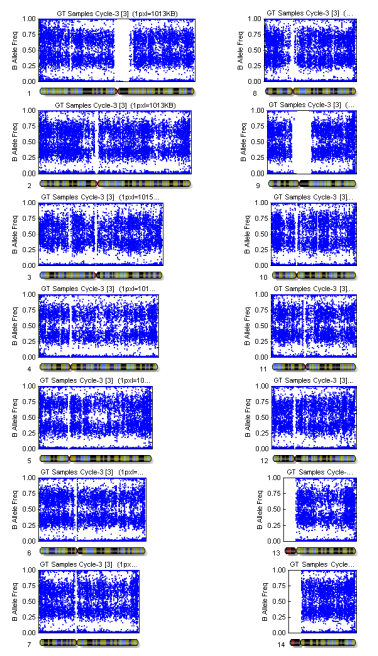

틀

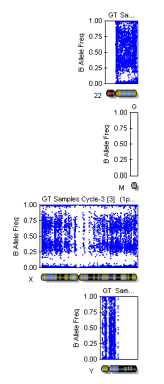

Cycle 5-2
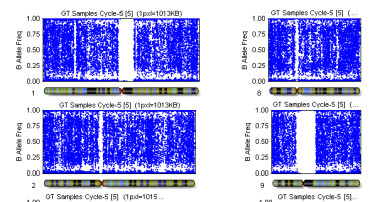

iil
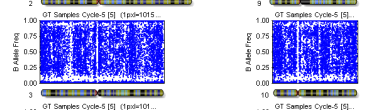

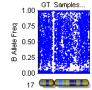
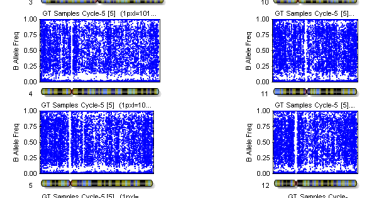

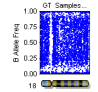
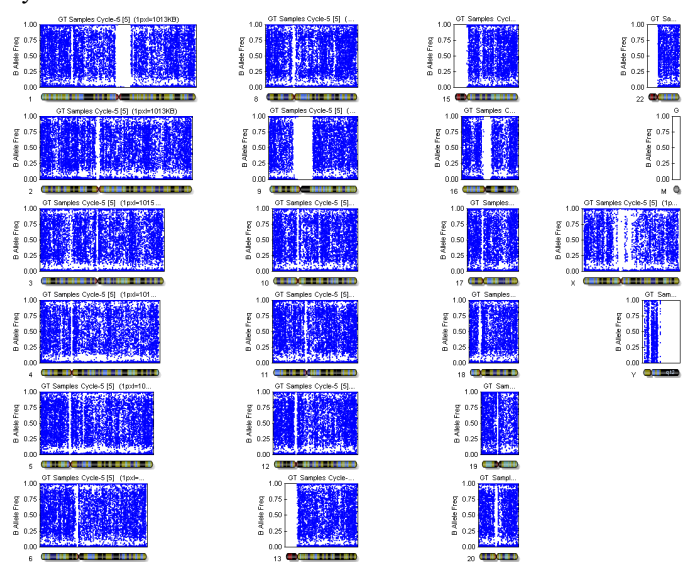

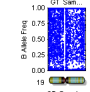

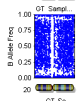
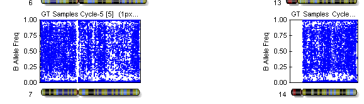

是

Figure 1: Triploidy detection during PGD/S using SNP array. Case 1-3: arr $(1-22) \times 3$, (X) $\times 3$; Case 2-1: arr $(1-22) \times 3$, (X) $\times 3$; Case 3-1: $\operatorname{arr}(1-22) \times 3,(\mathrm{X}) \times 3$; Case 4-3: $\operatorname{arr}(1-22) \times 3,(\mathrm{X}) \times 3 ;$ Case $5-2$ : $\operatorname{arr}(1-22) \times 3,(\mathrm{X}) \times 3$. 
Table 2: Comprehensive comparison of MDA based SNP array and MALBAC-NGS

\begin{tabular}{|c|c|c|}
\hline ID. & SNP array karyotype & NGS Karyotype \\
\hline 1 & arr $21($ pter $\rightarrow$ qter $) \times 3,(X) \times 2$ & $47, X X,+21(\times 3)$ \\
\hline 2 & arr $10($ pter $\rightarrow$ qter $) \times 3[\operatorname{mos} 20],(X) \times 2$ & $46, X X,+10 q(q 21.3 \rightarrow q t e r, \sim 64 M, \times 3, \operatorname{mos}, \sim 40 \%)$ \\
\hline 3 & arr $14($ pter $\rightarrow$ qter $) \times 3,(X) \times 2$ & $\begin{array}{c}47, \mathrm{XX},+4 \mathrm{q}(\mathrm{q} 28.1 \rightarrow \mathrm{q} 32.2, \sim 38 \mathrm{M}, \times 3, \mathrm{~m} \\
\text { os }),+14(\times 3),-17(\mathrm{p} 11.2 \rightarrow \mathrm{q} 21.33, \sim 30 \mathrm{M}, \times 1, \mathrm{mos}),- \\
19 \mathrm{q}(\mathrm{q} 13.11 \rightarrow \mathrm{qter}, \sim 23 \mathrm{M}, \times 1, \mathrm{mos})\end{array}$ \\
\hline 4 & $\operatorname{arr}(1-22) \times 2,(X) \times 2$ & $46, \mathrm{XX}$ \\
\hline 5 & $\operatorname{arr}(1-22) \times 2,(X) \times 2$ & $46, \mathrm{XX}$ \\
\hline 6 & $\begin{array}{l}\operatorname{arr} 21(\text { pter } \rightarrow \text { qter }) \times 1, \text { UPD }(6 \mathrm{p} 21.32 \rightarrow 6 \mathrm{p} 22.1) \\
\quad(27226965-35943151) \times 2 \mathrm{hmz},(\mathrm{XY}) \times 1\end{array}$ & $45, X Y,-21(\times 1)$ \\
\hline 7 & $\operatorname{arr}(1-22) \times 2,(X) \times 2$ & $46, \mathrm{XX}$ \\
\hline 8 & arr $16($ pter $\rightarrow$ qter $) \times 3,(X) \times 2$ & $47, \mathrm{XX},+16(\times 3)$ \\
\hline 9 & arr $20($ pter $\rightarrow$ qter $) \times 3,(X) \times 2$ & $47, \mathrm{XX},+20(\times 3)$ \\
\hline 10 & $\begin{array}{c}\operatorname{arr} 8 \mathrm{p}(\mathrm{pter} \rightarrow \mathrm{p} 12) \times 1,8 \mathrm{q}+[40] / 8 \mathrm{p}(\mathrm{pter} \rightarrow \mathrm{p} 12) \times 1,( \\
\mathrm{XY}) \times 1\end{array}$ & $46, X Y,-8 p($ pter $\rightarrow p 12, \sim 32 \mathrm{M}, \times 1)$ \\
\hline 11 & arr $14($ pter $\rightarrow$ qter $) \times 3,(X Y) \times 1$ & $47, \mathrm{XY},+14(\times 3)$ \\
\hline 12 & $\operatorname{arr} 4($ pter $\rightarrow$ qter $) \times 3,(X) \times 2$ & $47, \mathrm{XX},+4(\times 3)$ \\
\hline 13 & arr $7($ pter $\rightarrow$ qter $) \times 3,(X Y) \times 1$ & $47, \mathrm{XY},+7(\times 3)$ \\
\hline 14 & arr $16($ pter $\rightarrow$ qter $) \times 3,(X Y) \times 1$ & $47, \mathrm{XY},+16(\times 3)$ \\
\hline 15 & arr $16($ pter $\rightarrow$ qter $) \times 3,(X Y) \times 1$ & $47, \mathrm{XY},+16(\times 3)$ \\
\hline 16 & $\operatorname{arr}(1-22) \times 2,(X Y) \times 1$ & $46, X Y$ \\
\hline 17 & $\begin{array}{c}46, X X, \operatorname{dup}(1)(\mathrm{q} 32.1 \rightarrow \mathrm{qter})[100], 12 \mathrm{p}+[15], \operatorname{dup}(18) \\
(\mathrm{q} 21.32 \rightarrow \mathrm{qter})[20]\end{array}$ & $\begin{aligned} 46, X X,-1 \mathrm{q}(\mathrm{q} 32.2 & \rightarrow \mathrm{qter}, \sim 38 \mathrm{M}, \times 1, \mathrm{mos}),+18 \mathrm{q}(\mathrm{q} 21.31 \\
& \rightarrow \mathrm{qter}, \sim 21 \mathrm{M}, \times 3, \mathrm{mos})\end{aligned}$ \\
\hline 18 & arr $1 \mathrm{q}(\mathrm{q} 44) \times 1,8 \mathrm{q}(\mathrm{q} 24.22 \rightarrow \mathrm{qter}) \times 3,(\mathrm{XY}) \times 1$ & $46, X Y,+8 q(q 24.21 \rightarrow$ qter, $\sim 15 \mathrm{M}, \times 3)$ \\
\hline 19 & arr $8($ pter $\rightarrow$ qter $) \times 3,20($ pter $\rightarrow$ qter $) \times 3,(X) \times 2$ & $48, X X,+18(\times 3),+20(\times 3)$ \\
\hline 20 & $\operatorname{arr}(1-22) \times 2,(X Y) \times 1$ & $46, X Y$ \\
\hline 21 & $\begin{array}{c}\text { arr } 22(\text { pter } \rightarrow \text { qter }) \times 3,20(\text { pter } \rightarrow \text { qter }) \times 3[18] / 20(\text { pter } \rightarrow \\
\text { qter }) \times 3[82](X Y) \times 1\end{array}$ & $47, X Y,+22(\times 3)$ \\
\hline 22 & arr $8 \mathrm{p}(\mathrm{p} 22 \rightarrow \mathrm{pter}) \times 1,(\mathrm{X}) \times 2$ & $46, X X,-8 p(p t e r \rightarrow p 23.1, \sim 12 \mathrm{M}, \times 1)$ \\
\hline 23 & arr $16($ pter $\rightarrow$ qter $) \times 3,(X Y) \times 1$ & $47, \mathrm{XY},+16(\times 3)$ \\
\hline 24 & $\operatorname{arr} 15(\mathrm{pter} \rightarrow \mathrm{qter}),(\mathrm{X}$ & $47, \mathrm{XX},+15(\times 3)$ \\
\hline 25 & arr $15(\mathrm{q} 24.1$-qter $) \times 3,(X Y) \times 1$ & $46, X Y,+15 q(q 24.1 \rightarrow$ qter, $\sim 28 \mathrm{M}, \times 3)$ \\
\hline 26 & $\operatorname{arr} 22($ pter $\rightarrow$ qter $) \times 3,(X Y) \times 1$ & $47, \mathrm{XY},+22(\times 3)$ \\
\hline 27 & arr $3($ pter $\rightarrow$ qter $) \times 3,(X Y) \times 1$ & $47, \mathrm{XY},+3(\times 3)$ \\
\hline 28 & $\operatorname{arr}(1-22) \times 2,(X) \times 2$ & $46, \mathrm{XX},+8 \mathrm{q}(\mathrm{q} 24.13 \rightarrow \mathrm{qter}, \sim 20 \mathrm{M}, \times 3, \mathrm{mos}, \sim 40 \%)$ \\
\hline 29 & arr $13($ pter $\rightarrow$ qter $) \times 3,22($ pter $\rightarrow$ qter $) \times 3,(X Y) \times 1$ & $47, \mathrm{XY},+13(\times 3)$ \\
\hline 30 & arr $18($ pter $\rightarrow$ qter $) \times 3,(X Y) \times 1$ & $47, \mathrm{XY},+18(\times 3)$ \\
\hline 31 & arr $16($ pter $\rightarrow$ qter $) \times 3,(X Y) \times 1$ & $47, \mathrm{XY},+16(\times 3)$ \\
\hline 32 & arr $19(\mathrm{p} 12)(22130311-23202379) \times 3,(\mathrm{X}) \times 2$ & $46, X X,+13 q(q 31.1 \rightarrow q t e r, \sim 30 M, \times 3, \operatorname{mos}, \sim 30 \%)$ \\
\hline 33 & arr $11 \mathrm{q}(\mathrm{q} 22.3 \rightarrow \mathrm{q} 25) \times 3,11 \mathrm{q}(\mathrm{q} 25) \times 1,(\mathrm{XY}) \times 1$ & $46, X Y,+11 q(q 22.3 \rightarrow q 24.2, \sim 19 M, \times 3)$ \\
\hline 34 & arr $22($ pter $\rightarrow$ qter $) \times 3,(X) \times 2$ & $47, X X,+22(\times 3)$ \\
\hline 35 & arr $22($ pter $\rightarrow$ qter $) \times 3,(X) \times 2$ & $47, \mathrm{XX},+22(\times 3)$ \\
\hline 36 & arr 16 arr $16(\mathrm{pter} \rightarrow \mathrm{qter}) \times 3,(\mathrm{X}) \times 2$ & $47, X X,+16(\times 3)$ \\
\hline 37 & $\operatorname{arr} 5 \mathrm{q}(\mathrm{q} 23.1 \rightarrow \mathrm{qter}) \times 3,10(\mathrm{q} 26.2 \rightarrow \mathrm{q} 26.3) \times 1,(\mathrm{X}) \times 2$ & $46, X X,+5 q(q 22.3 \rightarrow$ qter $, \sim 65 \mathrm{M}, \times 3)$ \\
\hline
\end{tabular}

(Continued) 


\begin{tabular}{|c|c|c|}
\hline ID. & SNP array karyotype & NGS Karyotype \\
\hline 38 & arr $6($ pter $\rightarrow$ qter $) \times 3,(X) \times 2$ & $47, \mathrm{XX},+6(\times 3)$ \\
\hline 39 & arr $13($ pter $\rightarrow$ qter $) \times 3,(X Y) \times 1$ & $47, X Y,+13(\times 3)$ \\
\hline 40 & arr $16($ pter $\rightarrow$ qter $) \times 3,(X Y) \times 1$ & $47, X Y,+16(\times 3)$ \\
\hline 41 & arr $18(\mathrm{pter} \rightarrow \mathrm{qter}) \times 3,(\mathrm{X}) \times 2$ & $47, \mathrm{XX},+18(\times 3)$ \\
\hline 42 & $\begin{array}{c}46, X X, \operatorname{dup}(8)(\mathrm{q} 12.1 \rightarrow \mathrm{q} 21.1) 30 \%, \operatorname{dup}(19) \\
(\mathrm{q} 13.12 \rightarrow \mathrm{qter}) 30 \%, \operatorname{dup}(18)(\mathrm{q} 21.2 \rightarrow \mathrm{q} 22.1), \operatorname{del}(18) \\
(\mathrm{q} 22.1 \rightarrow \mathrm{qter})\end{array}$ & $\begin{aligned} & 46, X X,+18 \mathrm{q}(\mathrm{q} 11.2 \rightarrow \mathrm{q} 21.2, \sim 28 \mathrm{M}, \times 3, \mathrm{mos}),- \\
& 18 \mathrm{q}(\mathrm{q} 21.32 \rightarrow \mathrm{qter}, \sim 20 \mathrm{M}, \times 1)\end{aligned}$ \\
\hline 43 & arr $22(\mathrm{pter} \rightarrow \mathrm{qter}) \times 3,(\mathrm{X}) \times 2$ & $47, X X,+22(\times 3)$ \\
\hline 44 & arr $4 \mathrm{q}(\mathrm{q} 28.3 \rightarrow \mathrm{qter}) \times 3[\operatorname{mos} 15],(\mathrm{X}) \times 2$ & $46, \mathrm{XX}, 4 \mathrm{q}(\mathrm{q} 28.3 \rightarrow \mathrm{qter}) \times 3[\operatorname{mos} 30]$ \\
\hline 45 & arr $6($ pter $\rightarrow$ qter $) \times 3,(X) \times 2$ & $47, \mathrm{XX},+6(\times 3),-13 \mathrm{q}(\mathrm{q} 14.3 \rightarrow \mathrm{q} 22.1, \sim 21 \mathrm{M}, \times 1, \mathrm{mos})$ \\
\hline 46 & arr $16(\mathrm{pter} \rightarrow \mathrm{qter}) \times 3,(\mathrm{X}) \times 2$ & $47, \mathrm{XX},+16(\times 3)$ \\
\hline
\end{tabular}

A

D

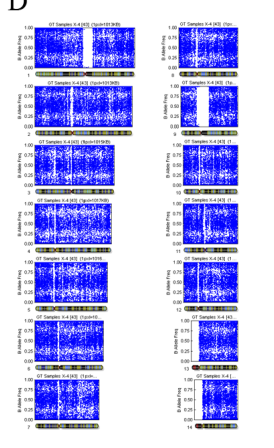

G

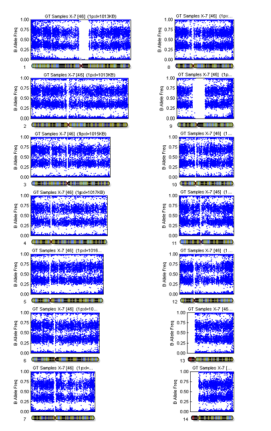

$\mathrm{B}$
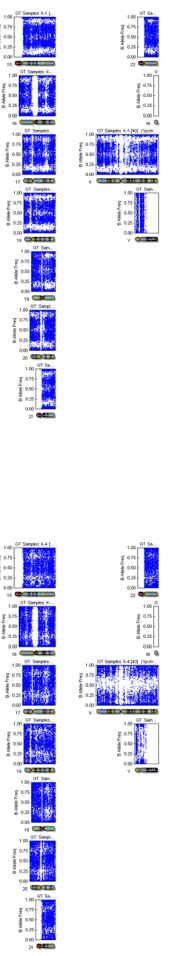

E
B
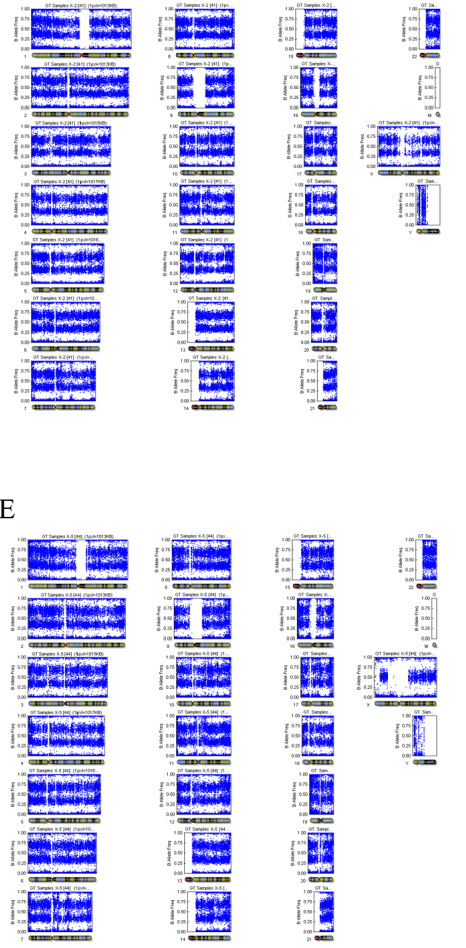

C

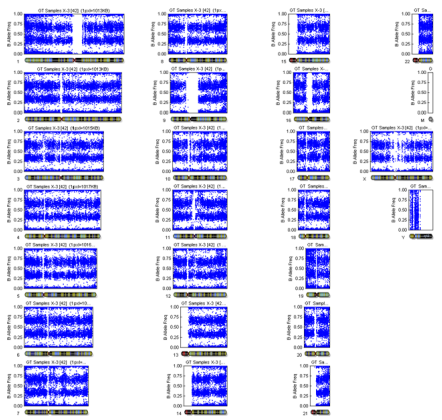

$\mathrm{F}$

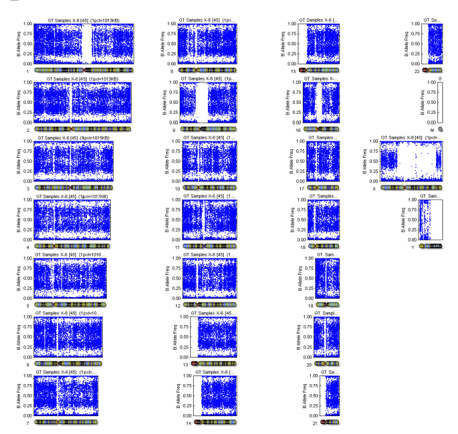

$\mathrm{H}$

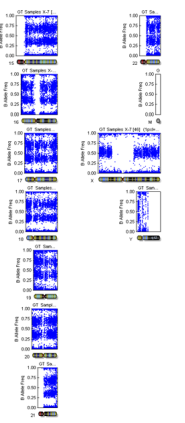

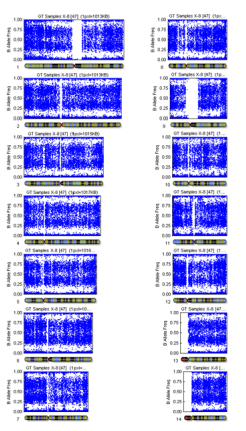

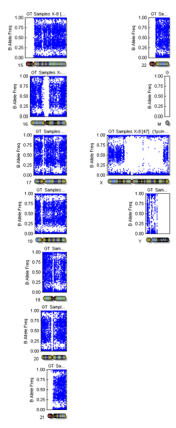

I

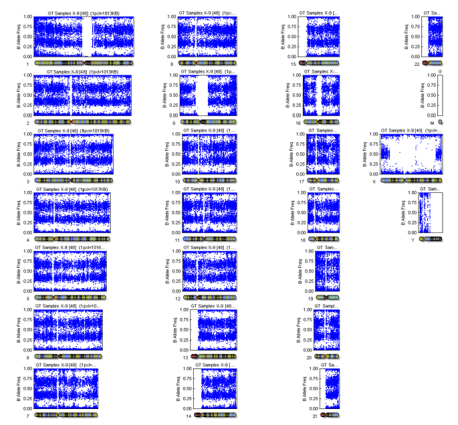

Figure 2: Three types of triploid identification of chorionic tissues using MDA based SNP array on single cell level. A-C. $\operatorname{arr}(1-22) \times 3,(X) \times 3$; D. 69,XYY, E-I. 69, XXY. 
widely in clinical [19], which had been proved to increase the pregnancy rate of chromosome structure abnormality carrier patients, advanced maternal age, mendelian diseases and recurrent miscarriage patients [17, 29, 30]. Previous data showed that PGD and PGS were good strategy for single embryo transfer which would reduce the multiple pregnancy rate without affecting the clinical outcomes and take baby home rate [31]. The accuracy and coverage of PGD and PGS technology are critical factors for its clinical applications, such as all aneuploidy kayotypes were successfully detected. SNP-array PGD was reported that may increase the clinical pregnancy outcome of translocation carriers [18]. However, only matched cohort studies relating to patients of advanced maternal age, recurrent miscarriage and implantation failure, limiting the ability to draw meaningful conclusions [32]. Triploidy as an abnormal embryo karyotype can occur at low rate in human spontaneous conception and IVF, it is negative correlation with pregnancy rate in IVF [12]. In our study, our data showed that both SNP array and NGS were sensitive to chromosome duplication, deletion and mosaic, while NGS could not identify uniparental disomy and not sensitive to triploidy.

In our study, both self and reference genome correction were used to analyze the NGS data of triploid DNA of chorionic tissues, and we found these two methods could not identify triploidy. The present bioinformatics algorithms mainly calculate the reads
Self correction

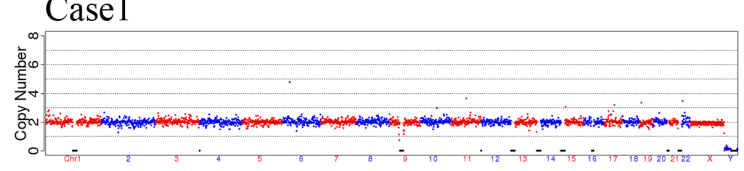

Case2

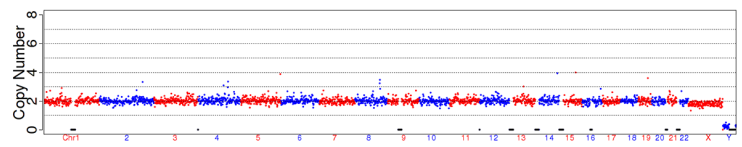

Case 3

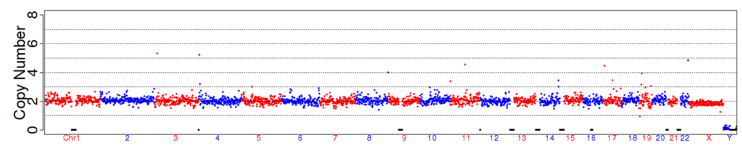

Case 4

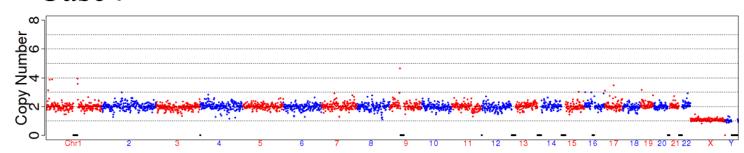

Case 5

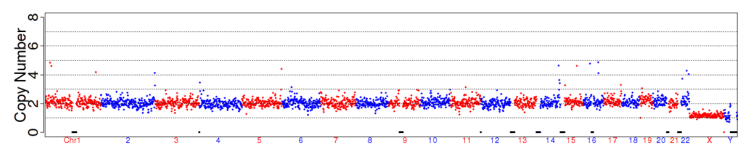

Case 7

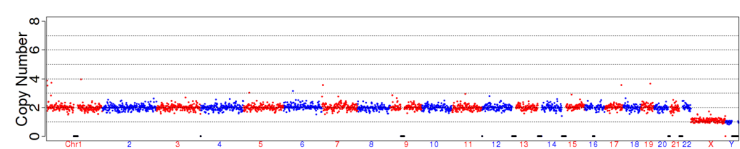

Case 8

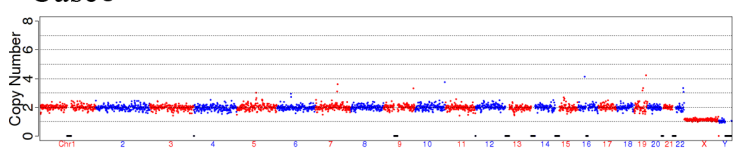

Case9

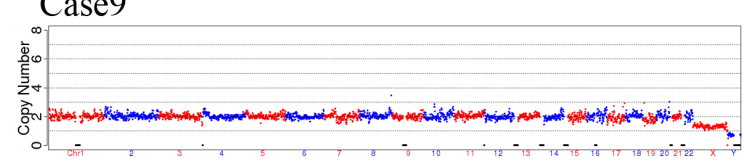

Reference genome correction
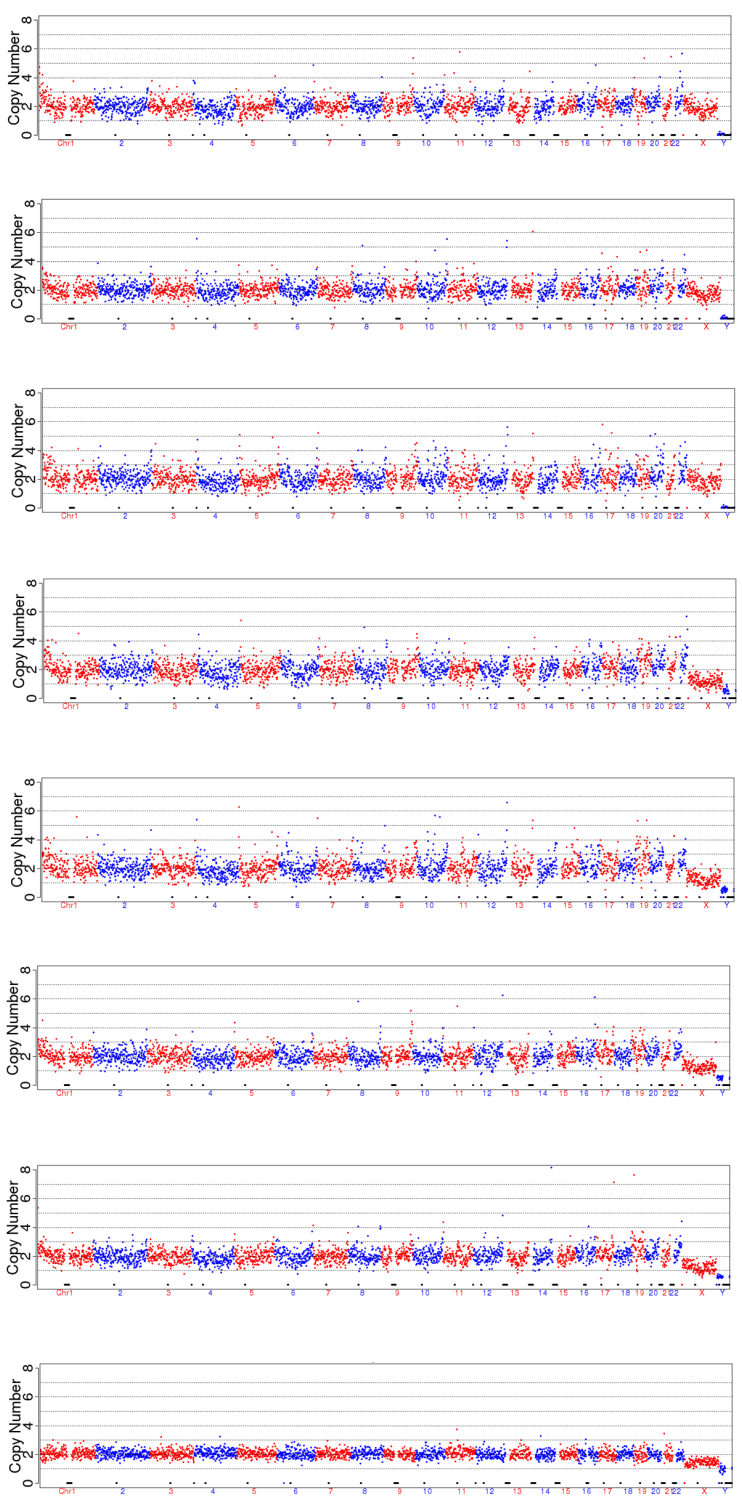

Figure 3: Self and reference genome correction of triploid chorionic tissue using NGS. 
falling into the "continues windows" usually ranging from $1 \mathrm{~K}$ to $1 \mathrm{M}$ on each chromosome, it is able to identify the reads number of the chromosome deletion, duplication and mosaic as shown in our study, but it ignores the SNPs and could not detect triploidy. Triploidy usually leads to the SNPs three nucleotides heterozygosis and the homozygous/heterozygosis was 1:2, SNP array well distinguishes the SNPs and well detects the triploidy even after the single cell whole genome amplification, while NGS needs to sequence deeper and much powerful bioinformatics algorisms to analyze the data. Although the NGS is much more powerful on detection micro duplication and deletion, it is weaker than SNP on detection triplody. SNP-based approach detected the relative distributions of alleles at polymorphic loci and does not require a reference chromosome for comparison [28]. Although MALBAC has increased the whole genome coverage rate, the triploidy still enlarge allele dropout rate for every chromosome of triploidy has same frequency to be amplification. Together with the genome recombination of triploidy will increase the difficulty of identification of triploidy. NATUS algorithm was used to distinguish the triploidy fetus using cfDNA from maternal blood, however it could not identify the SNPs precisely, for the allele dropout of single cell amplification [28]. Further study and strategies are essential to be developed to identify the triploidy using NGS. Time-lapse cooperated with NGS would be a better solution to decrease the insensitivity to triploid.

\section{MATERIALS AND METHODS}

\section{PGD and PGS patients controlled ovarian stimulation and embryos biopsy}

The controlled ovarian stimulation (COS) of all the patients underwent PGD and PGS were carried out in a long protocol, GnRH analogues was used for pituitary desensitisation, together with human menopausal gonadotrophins (hMG) or recombinant FSH. The starting dose of gonadotrophins for PGD and PGS patients was determined according to the patient's age, BMI and/or previous response to ovarian stimulation (range from 75 to $300 \mathrm{IU}$ QD). Human chorionic gonadotrophin (hCG) was administered when at least $60 \%$ follicles above 16 $\mathrm{mm}$ mean diameter and the biggest under $22 \mathrm{~mm}$ mean diameter were seen when transvaginal ultrasound scan. Transvaginal ultrasound-guided and vacuum takeoff oocytes collection was scheduled $36 \mathrm{~h}$ after hCG administration. Regardless of the sperm quality, Intra Cytoplasmic Sperm Injection (ICSI) was performed rather than IVF to prevent DNA contamination with sperm and cumulus cell's DNA during PGD and PGS. Fertilization was assessed 17-20 h after ICSI and embryo cleavage was recorded every $24 \mathrm{~h}$. Embryo biopsy was performed on day 5 or 6 at blastocyst stage. All patients were informed consents in our study [33].

\section{Single cell multiple displacement amplification (MDA) and SNP array}

Multiple Displacement Amplification (MDA) was used for single cell amplification in SNP array. Briefly, REPLI-g Single Cell Kit (QIAGEN, 150345) was used for single cell amplification, single cell was seeding in $4.5 \mu \mathrm{l}$ phosphate-buffered saline, and then was lysised using $3 \mu \mathrm{l}$ DTT and DLB in incubator for $10 \mathrm{~min}$ at $65^{\circ} \mathrm{C}$. After incubation, $3 \mu$ l Stop Solution was added in the mix. The amplification mix was prepared and $40 \mu$ including $2 \mu 1$ REPLI-g sc DNA polymerase and $29 \mu$ l REPLI-g sc Reaction Buffer was added to each tube. Then the mixture was incubated in incubator for $8 \mathrm{~h}$ at $30^{\circ} \mathrm{C}$ following $65^{\circ} \mathrm{C}$ for $3 \mathrm{~min}$ to inactivate the REPLI-g sc DNA polymerase. The single cell amplification mix was stored at $-20^{\circ} \mathrm{C}$ until for SNP array. The DNA of previous triploidy detected using SNP was diluted and amplification as described above. All the procedures were under the direction of Illumina human cyto12 microarray with minor modification in our center.

\section{Single cell multiple annealing and looping based amplification cycles (MALBAC) and next generation sequencing (NGS)}

Multiple Annealing and Looping Based Amplification Cycles (MALBAC) was used for whole genome amplification then high throughput sequencing. The DNA from previously SNP array results were quantified again with Qubit and then were diluted to 10pg for single cell amplification using YK015CHR (Yikon Genomics). Firstly the DNA was diluted into $4.5 \mu \mathrm{l}$ lysis buffer and lysis enzyme, the amplification products were fragmentation and ligated adapter, then PCR and magnetic purification according to the user manual. The barcoded DNA was sequenced using Hiseq 2500 Rapid $1 \times 50$ mode in our center.

\section{Single cell and multi cell SNP array data analysis}

The SNP array data was analyzed using GenomeStudio Software (2011, Illumina). B allele frequency and $\log \mathrm{R}$ ratio was used to analyzed the genotype. The copy number variance was called using KaryoStudio Software v1.4. The uniparental disomy was reported by both KaryoStudio and GenomeStudio.

\section{NGS data analysis using self correction and reference karyotype}

The raw data (in.bcl format) was demultiplexed and converted to FASTQ format using a perl script 
configureBclToFastq.pl in CASAVA 1.8.4 package based on the sample-sheet information. Illumina adaptors, low quality bases (bases with quality score less than 20) and MALBAC primers were removed from the FASTQ file using Trimmomatic [34].

High quality reads were mapped to hg19 reference genome using BWA with default parameters [35]. The mapped reads were sorted and converted to binary format using SamtoBam.jar in Picard package. Unique mapped reads were extract from the alignment reads (.bam file). Then the whole reference genome was divided into nonoverlapping observation windows (bins) with size of $1 \mathrm{Mb}$. Reads number, GC content were calculated in each bin. GC bias correction was processed for every $1 \% \mathrm{GC}$ content. The GC corrected relative reads number (RRN) of each bin was corrected by the reference training set [36]. 500 normal chromosome samples were prepared as reference training set, as well as self-correction were used to analyze the data. We use R programming language to graph the final RRN of each bin to visualize copy number variations.

\section{ACKNOWLEDGMENTS}

The authors would like to thank the Nurses of The Centre for Reproductive Medicine, their help are most grateful.

\section{CONFLICTS OF INTEREST}

The authors have declared that no competing interests exist.

\section{GRANT SUPPORT}

This work was supported by the National Natural Science Foundation (31271605 to Yingpu Sun), the National Natural Science Foundation (31501205 to Jiawei $\mathrm{Xu}$ ) the Youth Innovation Fund of the First Affiliated Hospital of Zhengzhou University (to Jiawei $\mathrm{Xu}$ and Wenbin Niu), and their supports are gratefully acknowledged.

\section{REFERENCES}

1. Doshi N, Surti U, Szulman AE. Morphologic anomalies in triploid liveborn fetuses. Hum Pathol. 1983; 14:716-723.

2. McFadden DE, Jiang $\mathrm{R}$, Langlois $\mathrm{S}$, Robinson WP. Dispermy--origin of diandric triploidy: brief communication. Hum Reprod. 2002; 17:3037-3038.

3. McFadden DE, Kwong LC, Yam IY, Langlois S. Parental origin of triploidy in human fetuses: evidence for genomic imprinting. Hum Genet. 1993; 92:465-469.

4. McFadden DE, Langlois S. Parental and meiotic origin of triploidy in the embryonic and fetal periods. Clin Genet. 2000; 58:192-200.
5. Redline RW, Hassold T, Zaragoza MV. Prevalence of the partial molar phenotype in triploidy of maternal and paternal origin. Hum Pathol. 1998; 29:505-511.

6. Jacobs PA, Szulman AE, Funkhouser J, Matsuura JS, Wilson CC. Human triploidy: relationship between parental origin of the additional haploid complement and development of partial hydatidiform mole. Ann Hum Genet. 1982; 46:223-231.

7. McFadden DE, Robinson WP. Phenotype of triploid embryos. J Med Genet. 2006; 43:609-612.

8. Jun SH, O'Leary T, Jackson KV, Racowsky C. Benefit of intracytoplasmic sperm injection in patients with a high incidence of triploidy in a prior in vitro fertilization cycle. Fertil Steril. 2006; 86:825-829.

9. Egozcue S, Blanco J, Vidal F, Egozcue J. Diploid sperm and the origin of triploidy. Hum Reprod. 2002; 17:5-7.

10. Macas E, Imthurn B, Keller PJ. Increased incidence of numerical chromosome abnormalities in spermatozoa injected into human oocytes by ICSI. Hum Reprod. 2001; 16:115-120.

11. Rosen MP, Shen S, Dobson AT, Fujimoto VY, McCulloch CE, Cedars MI. Triploidy formation after intracytoplasmic sperm injection may be a surrogate marker for implantation. Fertil Steril. 2006; 85:384-390.

12. Dayal MB, Gindoff PR, Sarhan A, Dubey A, Peak D, Frankfurter D. Effects of triploidy after intracytoplasmic sperm injection on in vitro fertilization cycle outcome. Fertil Steril. 2009; 91:101-105.

13. Thornhill AR, deDie-Smulders CE, Geraedts JP, Harper JC, Harton GL, Lavery SA, Moutou C, Robinson MD, Schmutzler AG, Scriven PN, Sermon KD, Wilton L, Consortium EP. ESHRE PGD Consortium 'Best practice guidelines for clinical preimplantation genetic diagnosis (PGD) and preimplantation genetic screening (PGS)'. Hum Reprod. 2005; 20:35-48.

14. Grossmann M, Calafell JM, Brandy N, Vanrell JA, Rubio C, Pellicer A, Egozcue J, Vidal F, Santalo J. Origin of tripronucleate zygotes after intracytoplasmic sperm injection. Hum Reprod. 1997; 12:2762-2765.

15. Sachs AR, Politch JA, Jackson KV, Racowsky C, Hornstein $\mathrm{MD}$, Ginsburg ES. Factors associated with the formation of triploid zygotes after intracytoplasmic sperm injection. Fertil Steril. 2000; 73:1109-1114.

16. Harper JC, Wilton L, Traeger-Synodinos J, Goossens V, Moutou C, SenGupta SB, Pehlivan Budak T, Renwick P, De Rycke M, Geraedts JP, Harton G. The ESHRE PGD Consortium: 10 years of data collection. Hum Reprod Update. 2012; 18:234-247.

17. Tan Y, Yin X, Zhang S, Jiang H, Tan K, Li J, Xiong B, Gong F, Zhang C, Pan X, Chen F, Chen S, Gong C, Lu C, Luo $\mathrm{K}, \mathrm{Gu} \mathrm{Y}$, et al. Clinical outcome of preimplantation genetic diagnosis and screening using next generation sequencing. Gigascience. 2014; 3:30. 
18. Tan YQ, Tan K, Zhang SP, Gong F, Cheng DH, Xiong B, Lu CF, Tang XC, Luo KL, Lin G, Lu GX. Single-nucleotide polymorphism microarray-based preimplantation genetic diagnosis is likely to improve the clinical outcome for translocation carriers. Hum Reprod. 2013; 28:2581-2592.

19. Dahdouh EM, Balayla J, Audibert F, Genetics C, Wilson RD, Audibert F, Brock JA, Campagnolo C, Carroll J, Chong K, Gagnon A, Johnson JA, MacDonald W, Okun N, Pastuck M, Vallee-Pouliot K. Technical Update: Preimplantation Genetic Diagnosis and Screening. J Obstet Gynaecol Can. 2015; 37:451-463.

20. van Uum CM, Stevens SJ, Dreesen JC, Drusedau M, Smeets HJ, Hollanders-Crombach B, Die-Smulders CE, Geraedts JP, Engelen JJ, Coonen E. SNP array-based copy number and genotype analyses for preimplantation genetic diagnosis of human unbalanced translocations. Eur J Hum Genet. 2012; 20:938-944.

21. Munne S. Preimplantation genetic diagnosis for aneuploidy and translocations using array comparative genomic hybridization. Curr Genomics. 2012; 13:463-470.

22. Martin J, Cervero A, Mir P, Martinez-Conejero JA, Pellicer A, Simon C. The impact of next-generation sequencing technology on preimplantation genetic diagnosis and screening. Fertil Steril. 2013; 99:1054-1061 e1053.

23. Treff NR, Fedick A, Tao X, Devkota B, Taylor D, Scott RT, Jr. Evaluation of targeted next-generation sequencing-based preimplantation genetic diagnosis of monogenic disease. Fertil Steril. 2013; 99:1377-1384 e1376.

24. Yan L, Huang L, Xu L, Huang J, Ma F, Zhu X, Tang Y, Liu M, Lian Y, Liu P, Li R, Lu S, Tang F, Qiao J, Xie XS. Live births after simultaneous avoidance of monogenic diseases and chromosome abnormality by next-generation sequencing with linkage analyses. Proc Natl Acad Sci U S A. 2015 ; 112:15964-15969.

25. Huang L, Ma F, Chapman A, Lu S, Xie XS. SingleCell Whole-Genome Amplification and Sequencing: Methodology and Applications. Annu Rev Genomics Hum Genet. 2015; 16:79-102.

26. Chen M, Song P, Zou D, Hu X, Zhao S, Gao S, Ling F. Comparison of multiple displacement amplification (MDA) and multiple annealing and looping-based amplification cycles (MALBAC) in single-cell sequencing. PLoS One. 2014; 9:e114520.

27. Curnow KJ, Wilkins-Haug L, Ryan A, Kirkizlar E, Stosic M, Hall MP, Sigurjonsson S, Demko Z, Rabinowitz M,
Gross SJ. Detection of triploid, molar, and vanishing twin pregnancies by a single-nucleotide polymorphism-based noninvasive prenatal test. Am J Obstet Gynecol. 2015; 212:79 e 71-79.

28. Nicolaides KH, Syngelaki A, del Mar Gil M, Quezada MS, Zinevich Y. Prenatal detection of fetal triploidy from cellfree DNA testing in maternal blood. Fetal Diagn Ther. 2014; 35:212-217.

29. Moutou C, Goossens V, Coonen E, De Rycke M, Kokkali G, Renwick P, SenGupta SB, Vesela K, Traeger-Synodinos J. ESHRE PGD Consortium data collection XII: cycles from January to December 2009 with pregnancy follow-up to October 2010. Hum Reprod. 2014; 29:880-903.

30. Lukaszuk K, Pukszta S, Wells D, Cybulska C, Liss J, Plociennik L, Kuczynski W, Zabielska J. Routine use of next-generation sequencing for preimplantation genetic diagnosis of blastomeres obtained from embryos on day 3 in fresh in vitro fertilization cycles. Fertil Steril. 2015; 103:1031-1036.

31. Huang J, Li R, Lian Y, Chen L, Shi X, Qiao J, Liu P. Vitrified/warmed single blastocyst transfer in preimplantation genetic diagnosis/preimplantation genetic screening cycles. Int J Clin Exp Med. 2015; 8:21605-21610.

32. Lee E, Illingworth P, Wilton L, Chambers GM. The clinical effectiveness of preimplantation genetic diagnosis for aneuploidy in all 24 chromosomes (PGD-A): systematic review. Hum Reprod. 2015; 30:473-483.

33. Siristatidis C, Sergentanis TN, Kanavidis P, Trivella M, Sotiraki M, Mavromatis I, Psaltopoulou T, Skalkidou A, Petridou ET. Controlled ovarian hyperstimulation for IVF: impact on ovarian, endometrial and cervical cancer--a systematic review and meta-analysis. Hum Reprod Update. 2013; 19:105-123.

34. Bolger AM, Lohse M, Usadel B. Trimmomatic: a flexible trimmer for Illumina sequence data. Bioinformatics. 2014; 30:2114-2120.

35. Li H, Durbin R. Fast and accurate short read alignment with Burrows-Wheeler transform. Bioinformatics. 2009; 25:1754-1760.

36. Zhang C, Zhang C, Chen S, Yin X, Pan X, Lin G, Tan Y, Tan K, Xu Z, Hu P, Li X, Chen F, Xu X, Li Y, Zhang X, Jiang $\mathrm{H}$, et al. A single cell level based method for copy number variation analysis by low coverage massively parallel sequencing. PLoS One. 2013; 8:e54236. 